\title{
Relato
}

\section{Assistência à saúde integral do binômio mãe- filho no quinto dia de vida do bebê: relato de uma experiência}

Integral health assistance of the binomial mother-child on the fifth day of the baby's life: report of an experience

\section{Introdução}

O retorno ambulatorial nas primeiras semanas de vida do recém-nascido é de fundamental importância para garantir a saúde do mesmo, por meio de medidas de promoção à saúde, prevenção e diagnóstico precoce. Dessa forma, todo recém-nascido deveria ser visto pelo pediatra ou outro profissional de saúde entre o $3^{\circ}$ e o $5^{\circ}$ dia de vida, conforme recomenda o Ministério da Saúde (MS) em seu "Manual para utilização da Caderneta de Saúde da Criança (2005), o protocolo de atenção à criança na "Primeira Semana Saúde Integral" .

Dentre as orientações oferecidas durante a consulta na primeira semana de vida, a promoção do aleitamento materno é de grande importância, sendo considerada pela Organização Mundial de Saúde como uma das cinco ações básicas de saúde no combate à mortalidade infantil e na melhoria da condição de vida desta população ${ }^{1,2}$.

Com o objetivo de apoiar e promover o aleitamento materno no Brasil, criou-se a Iniciativa Unidade Amiga Básica da Amamentação (IUBAAM), seguindo a mesma orientação do Hospital Amigo da Criança da United Nations Children's Fund (UNICEF). Dentre os 10 passos para o sucesso do aleitamento, estão: ouvir as preocupações e dúvidas das gestantes e mães quanto à amamentação, fortalecendo sua autoconfiança; mostrar às gestantes e mães como amamentar e como manter a lactação, mesmo se estiverem ou vierem a ser separadas de seus filhos; encorajar a amamentação sob livre demanda; orientar todas as gestantes e mães sobre os riscos do uso de mamadeiras, bicos e chupetas ${ }^{3,4,5}$.

Apesar das ações de incentivo ao aleitamento materno em Juiz de Fora, a taxa de aleitamento materno exclusivo no quarto mês de vida, no refe-

\author{
Vivianne Weil Afonso', Bernardo Silveira de \\ Moraes Knopp ${ }^{2}$, José Nalon de Queiroz ${ }^{3}$, \\ Patrícia Gomes Pinto ${ }^{4}$, Ivana Lúcia Damásio \\ Moutinho ${ }^{5}$, José Carlos Coelho Magalhães ${ }^{6}$, Karla \\ Cunha Mendes7, Uiara Raiana Vargas de Castro \\ Oliveira Ribeiro ${ }^{8}$, Daniel Almeida do Valle ${ }^{9}$
}

\begin{abstract}
Resumo
O projeto "Assistência à saúde integral do binômio mãe-filho no quinto dia de vida do bebê" é pioneiro em Juiz de Fora e tem sido realizado desde 2005 por uma equipe multidisciplinar. Tem por objetivos principais: orientar a mãe acerca de cuidados com a sua saúde e de seu filho recém-nascido, detectar binômios com situação de risco para sua saúde encaminhando-os para atendimento especializado, estimular e orientar a puérpera para o aleitamento materno e mostrar-lhe a importância da utilização da Caderneta de Saúde da Criança além de introduzir o aluno de medicina no trabaIho ético em equipe e na medicina social.
\end{abstract}

Palavras-chaves: Aleitamento materno; Medicina Social; Puericultura

Área temática: Saúde

Linha de extensão: Saúde humana
1 Professora. UFJF. Email: vwafonso@yahoo.com.br
2 Professor. UFJF. Email: berknopp@yahoo.com.br
${ }^{3}$ Professor UFJF. Email: jnalon@bol.com.br
${ }^{4}$ Professora. UFJF. Email: patricia.gomes@edu.ufjf.br
${ }^{5}$ Professor. UFJF. Email: jctcoelho@gmail.com
${ }^{6}$ Professora UFJF. Email: ivanadamásio@terra.com.br
7 Enfermeira. Prefeitura Municipal de Juiz de Fora - Juiz de Fora, MG. Email:
vwafonso@yahoo.com.br
${ }^{8}$ Bolsista de extensão. UFJF. Email: uiarinha@yahoo.com.br
${ }^{9}$ Bolsista de extensão. UFJF. Email: almeida.valle@yahoo.com.br 
rido município, em 2002, estava em 20,8\%, resultado esse inferior à maioria das capitais brasileiras, exceto Cuiabá ${ }^{3,6}$. No Brasil, a maioria das crianças $(67,7 \%)$ inicia o aleitamento na primeira hora de vida, mas a manutenção de forma exclusiva até os 4 meses é aquém do desejado (51,29\%), com taxas ainda menores se considerada até os 6 meses de vida $(41 \%)^{3,7}$.

Como estratégia para aumentar os índices de amamentação em nosso município e orientar a saúde do binômio mãe-filho, realiza-se um projeto de extensão de educação em saúde da Universidade Federal de Juiz de Fora (UFJF), no Departamento de Saúde da Criança e do Adolescente (DSCA), através da parceria entre UFJF e o Sistema Único de Saúde (SUS), no qual a mãe que procura o local para realizar o teste do pezinho recebe orientação especializada por equipe multidisciplinar acerca de aleitamento materno e cuidados com sua saúde e de seu filho.

O DSCA é referência no município juiz-forano para realização do teste do pezinho pelo SUS, exame este obrigatório no Brasil para detecção de patologias como hipotireoidismo, fenilcetonúria, mucoviscidose e hemoglobinopatias ${ }^{8}$. Por mês são realizados aproximadamente 400 exames de Triagem Neonatal no DSCA.

Assim, propõe-se aqui descrever as atividades realizadas por este projeto de educação em saúde aos pais acerca de aleitamento materno e cuidados com o binômio mãe-filho, por equipe de profissionais de saúde, estudantes de medicina e residentes de pediatria da UFJF, na ocasião da realização do teste do pezinho em unidade de saúde de Juiz de Fora, Minas Gerais.

\section{Objetivos}

Este relato tem como objetivo descrever as atividades realizadas no projeto de extensão "Assistência à saúde integral do binômio mãe-filho no quinto dia de vida do bebê".

\section{Desenvolvimento}

O projeto de extensão "Assistência à saúde integral do binômio mãe-filho no quinto dia de vida do bebê" iniciou-se em 2005 e é desenvolvido por pediatras, equipe de enfermagem do DSCA, professores de pediatria, alunos de medicina e re- sidentes de pediatria da UFJF, na Unidade de Clinicas Especializadas, órgão do Departamento de Saúde da Criança e do Adolescente do SUS, localizado na rua Espírito Santo, em Juiz de Fora, MG. Nesse local funcionam o Serviço de Puericultura, Serviço de Atenção à Saúde do Adolescente, o Banco de Leite Humano, as salas de vacinação, de saúde bucal e de coleta de material para testes de triagem neonatal para hipotireoidismo, fenilcetonúria, mucoviscidose e hemoglobinopatias, e o serviço de referência em oftalmologia para crianças com alterações no teste do reflexo vermelho. Este projeto faz parte de outro maior denominado "Atendimento médico e estímulo ao aleitamento materno em Serviço Especial de Puericultura".

$O$ projeto tem como objetivo principal realizar a promoção da saúde e prevenção de doenças em recém-nascidos e suas mães, atendidos no DSCA, na ocasião da realização do teste do pezinho, orientando a mãe acerca de cuidados com a sua saúde e a de seu filho.

Além disso, propõe-se detectar mães e recém-nascidos em situação de risco para sua saúde, encaminhar para atendimento especializado as mães e recém-nascidos com algum critério de risco à saúde conforme orientações do MS, estimular e orientar a puérpera para o aleitamento materno, e orientar a família acerca da importância e utilização da Caderneta de Saúde da Criança.

Com esse projeto, permite-se aos alunos vivenciar os aspectos éticos da profissão e introduzi-los no trabalho em equipe interdisciplinar, bem como na medicina social, com troca de experiências entre profissionais da Instituição. $O$ aluno é também orientado quanto à assistência ao recém-nascido em relação ao aleitamento materno, aspectos da anamnese, exame físico e realização de receituário.

Os alunos de medicina fazem rodízio mensal e recebem treinamento inicial, pela coordenação do projeto, acerca da abordagem, sinais de risco de saúde para os binômios, aleitamento materno, vacinação e realização dos procedimentos relatados. Passam também por aprendizado no Banco de Leite Humano, sala de vacinação, teste do pezinho e do olhinho, ambulatórios de pediatria e puericultura.

As técnicas de enfermagem responsáveis pelo teste do pezinho recebem treinamento em aleitamento materno no Banco de Leite Humano assim como acerca dos sinais de risco à saúde com 
a coordenação do projeto. Realizam, ainda, a captação do binômio mãe-filho, alvo do projeto em todo o período de realização do teste do pezinho, de 8 às 17 horas, diariamente.

O acolhimento adequado é essencial em toda a entrevista: ficar ao lado da mãe, olhar em seus olhos quando necessário, pedir licença para escrever, evitar ser interrompido por outras pessoas ou telefonemas, deixá-la falar, fazendo reforço positivo a fim de que ela se sinta à vontade para expor suas dúvidas, evitar julgamentos e usar linguagem acessível. Esse tipo de atendimento é praticado por toda equipe e repassado aos alunos continuadamente ${ }^{9}$.

Atualmente, três professores e uma pediatra do serviço participam do atendimento todas as manhãs e uma tarde. Nos outros horários, existem pediatras no ambulatório de puericultura e pediatria que atendem as emergências quando necessário.

O projeto conta com a aprovação da direção do DSCA e com a cooperação de toda a equipe local para sua realização, o que demonstra a sua importância e viabilidade.

Segue-se a metodologia utilizada para a abordagem do binômio mãe-filho por ocasião do teste do pezinho neste projeto.

\section{Captação dos binômios de risco}

A captação dos binômios de risco ocorre em três momentos, para todos os binômios materno-infantis que irão se submeter ao teste de triagem neonatal no local: (1) abordagem pela equipe de enfermagem que realiza o teste do pezinho; (2) abordagem em grupo por pediatra e alunos da Faculdade de Medicina da UFJF no momento da realização de orientações acerca da saúde materno-infantil; (3) abordagem individual por alunos do estágio de medicina da UFJF e por um professor da Faculdade de Medicina da UFJF. No seu início, o projeto contava com a participação do residente de pediatria.

A equipe de saúde, ao detectar problemas com o binômio mãe-filho, conforme explicitado a seguir, encaminha o mesmo para a avaliação do pediatra.

\section{Critérios para escolha do binômio de risco}

A escolha dos binômios a serem assistidos segue, para o recém-nascido, os critérios de risco para crianças menores de 2 meses $^{1,10,11}$.
São considerados sinais de alerta: bebê "paradinho", muito sonolento, com dificuldade para acordar, convulsão, vômitos repetidos, pouca diurese, palidez, icterícia em membros, dificuldade para mamar, hipo ou hipertermia, umbigo com hiperemia, mau odor ou secreção. Em caso de anormalidade, encaminha-se ao pediatra ${ }^{1,10}$.

Para as puérperas, segue-se a orientação do Manual Técnico para o Pré-natal e Puerpério do Ministério da Saúde de 2006. De acordo com o referido manual, são considerados sinais de alerta e atenção: sangramento vaginal persistente vermelho vivo; mal cheiro dos lóquios; temperatura acima de $38^{\circ}$ ou mais em 2 tomadas seguidas com intervalo de 06 horas; aumento da sensibilidade; dor localizada; vermelhidão nas panturrilhas; dor ou aumento da sensibilidade abdominal ou pélvica; mamas doloridas, sensíveis ou área vermelha localizada; dor anormal na área da incisão, com ou sem secreção purulenta; micção dolorosa ${ }^{12}$.

Os binômios nos quais se identificou algum dos critérios descritos anteriormente são encaminhados para a avaliação de um pediatra, juntamente com alunos do curso de medicina, no próprio DSCA.

\section{Procedimentos na abordagem do binômio mãe-filho}

O pediatra, juntamente com os alunos de medicina, consulta e realiza os seguintes procedimentos nos binômios materno-infantil captados:

- Avaliação e orientação do uso da Caderneta de Saúde da Criança (ou Cartão de Saúde da Criança): anotam-se dados sobre o nascimento, desenvolvimento, crescimento e vacinação da criança, norteando seu preenchimento correto pelo profissional de saúde.

- Identificação de situações de risco para o recém-nascido ${ }^{1,10}$.

- Verificação da frequência das eliminações do recém-nascido.

- Realização de exame físico completo do recém-nascido, inclusive teste do olhinho.

- Avaliação de uma mamada (pega, posição, saída de leite, etc).

- Verificação se perda de peso está maior que a fisiológica: atenção e intervenção para corrigir problemas. 
- Orientação de higiene do coto umbilical.

- Verificação da história materna no Cartão de Pré-natal: exames do pré-natal, problemas de saúde, vacinação ${ }^{13}$.

- Encaminhamentos conforme a necessidade. Para a mãe: obstetra (no Pronto Atendimento Médico - PAM - Marechal), vacinação (no DSCA) e Banco de Leite Humano (DSCA). Para o RN: pediatra no DSCA ou Pronto Atendimento Infantil, laboratório para exames complementares se necessário (PAM Andradas), vacinas (DSCA), Teste do Pezinho (DSCA) e Teste da orelhinha (hospital de referência).

- Orientação do retorno posterior para reavaliação do binômio, conforme a necessidade e monitoramento do aleitamento materno neste período crítico, no próprio DSCA. Caso não seja identificada necessidade de acompanhamento deste binômio em um nível secundário de atenção à saúde, recomendar a marcação de consulta de rotina para o binômio em sua Unidade de Atenção Primária à Saúde.

A abordagem individual pelo estagiário de medicina segue os seguintes passos:

- Apresentação: "A Sra. está bem? E seu bebê?".

- Inspeção: procurar sinais de perigo descritos anteriormente e encaminhar ao pediatra se indicado ${ }^{1,10,11}$.

- A Caderneta de Saúde da Criança (ou Cartão da Criança): ressaltar sua importância, mostrando aos pais as páginas que o profissional de saúde deve preencher a cada consulta (dados da gravidez, parto, puerpério e nascimento, triagem neonatal, gráficos de crescimento, dados das consultas, vacinas) e as páginas destinadas a orientações para a família (desenvolvimento, prevenção de acidentes etc).

- Orientar o aleitamento materno - "O bebê mama bem?", encaminhando ao Banco de Leite Humano se necessário.

- Avaliar Cartão da Gestante - "E com a sra, tudo bem?", encaminhando se indi- cado (Instituto da Mulher, Banco de Leite Humano, vacina) $)^{12,13}$.

- Encaminhar para teste do pezinho, teste da orelhinha, orientação saúde bucal e marcação dos retornos, se necessário.

\section{Conclusão}

O projeto "Assistência à saúde integral do binômio mãe-filho no quinto dia de vida do bebê" é pioneiro em Juiz de Fora e tem sido realizado com muita dedicação por toda a equipe de saúde mostrando resultados animadores. Os binômios avaliados pela equipe são encaminhados em tempo hábil para a resolução dos casos patológicos ao mesmo tempo em que recebem orientações acerca dos cuidados com sua saúde, com ênfase ao aleitamento materno exclusivo. E para a multiplicação das ações do projeto, profissionais de saúde vão sendo continuamente habilitados para novos serviços.

É importante ressaltar que as avaliações realizadas pelo projeto não substituem a consulta de puericultura ou a consulta de puerpério, devendo essas serem realizadas conforme as recomendações das sociedades ${ }^{1,12}$.

Para os acadêmicos e demais profissionais de saúde envolvidos no projeto, o trabalho multi e interdisciplinar desenvolvido com bases éticas e sociais conduz a uma formação e prática em saúde mais humanizada e mais inserida no contexto social.

Esse tipo de programa pode ser desenvolvido por equipe de qualquer unidade de saúde que se sinta motivada, sendo o material necessário de pouca complexidade. O essencial é o desejo de querer contribuir para melhorar a educação para saúde das mães, com consequente melhora da qualidade de vida para seu filho e sua família.

\section{Contribuições dos Autores}

Uiara Raiana Vargas de Castro Oliveira Ribeiro e Daniel Almeida do Valle, alunos da faculdade de medicina - UFJF, participaram do projeto de extensão e da redação base do relato de experiência.

Bernardo Silveira de Moraes Knopp, José Nalon de Queiroz, Patrícia Gomes Pinto, Ivana Lúcia Damásio Moutinho, José Carlos Coelho 
Magalhães e Karla Cunha Mendes atuaram como co-orientadores do projeto de extensão, participando da elaboração e realização das atividades com o grupo de alunos.

Vivianne Weil Afonso foi responsável pela orientação do projeto de extensão e pela revisão do relato de experiência.

\section{Referências}

1. BRASIL. MINISTÉRIO DA SAÚDE. Manual para utilização da Caderneta de Saúde da Criança. 6. ed. Série A. Normas e Manuais Técnicos. Brasília, DF, 2005. 44 p.

2. BRASIL. MINISTÉRIO DA SAÚDE. Manual técnico para a Saúde da Criança: acompanhamento do crescimento e desenvolvimento infantil. Ministério da Saúde. Secretaria de Políticas de Saúde. Brasília: MS, 2002. 100 p.

3. BRASIL. Prevalência de Aleitamento Materno nas capitais brasileiras e Distrito Federal. Brasília: MS, 2001. 50 p.

4. ORGANIZAÇÃO MUNDIAL DE SAÚDE (OMS). OPAS. UNICEF. Manejo e promoção do Aleitamento Materno. Curso de 18 hs para equipes de maternidades. New York, 1993, 135 p.

5. ORGANIZAÇÃO MUNDIAL DE SAÚdE (OMS). OPAS. Saúde e Desenvolvimento da Criança. Evidências
Científicas dos Dez Passos para o Sucesso do Aleitamento Materno. Brasília: MS, 2001. 134 p.

6. AFONSO, Vivianne Weil. Prevalência de Aleitamento Materno em Juiz de Fora, MG. Tese de Mestrado no IMS UERJ/ UFJF, 2002. 127 p.

7. BRASIL. Prevalência de Aleitamento Materno nas capitais brasileiras e Distrito Federal. Brasília: MS, 2009. 103 p.

8. ARANHA, Maria Aparecida Figueiredo. Triagem neonatal. In: ESCOBAR, Ana Maria de Ulhôa; VALENTE, Maria Helena; GRISI, Sandra Josefina Ferraz Elero. A promoção da saúde na Infância. São Paulo: Ed. Manole, 2009, p. 122-38.

9. OLIVEIRA, Leda Maria Leal; TUNIN, Andréa Simoni Manarin; SILVA, Fernanda Cristina. Acolhimento: concepÇões, implicações no processo de trabalho e na atenção em saúde. Revista de Atenção Primária à Saúde. Juiz de Fora, v. 11, n. 4, p. 362-73, out/dez, 2008.

10. BRASIL. MINISTÉRIO DA SAÚDE. Caderneta de Saúde da Criança. 2007. Disponível em: <http://www.saúde. gov.br. Acesso em: 18 de abril de 2009.

11. BRASIL. MINISTÉRIO DA SAÚDE. Caderneta de Saúde da Criança. 2009. Disponível em: <http://www.saúde. gov.br>. Acesso em: 24 de setembro de 2010.

12. BRASIL. MINISTÉRIO DA SAÚDE. Manual Técnico para o Pré-natal e Puerpério. Brasília, DF, 2006. 162 p.

13. GOVERNO DO ESTADO. Minas Gerais. Cartilha da Gestante, GEMG, p.13, sd.

\section{Abstract}

The project "Integral health assistance of the binomial mother-child on the fifth day of the baby's life" is a pioneer initiative in Juiz de Fora and has been held since 2005 by a multidisciplinary team. Its main objectives are: to provide the mother guidance regarding health care for themselves and their baby; to detect binomials in health-risky situations to stimulate and to guide the puerperal towards mother breastfeeding and to demonstrate the importance of the utilization of the Child's Health Record; to introduce medical students to ethical team work and social medicine.

Keywords: Breastfeeding; Social Medicine; Puericulture 